\title{
Quality of family planning counseling among women attending prenatal care at a hospital in Addis Ababa, Ethiopia
}

\author{
Abel Teshome $^{1 *}$ | Malede Birara ${ }^{1}$ | Sarah D. Rominski ${ }^{2}$
}

${ }^{1}$ Department of Obstetrics and Gynecology, St. Paul's Hospital Millennium Medical College, Addis Ababa, Ethiopia

${ }^{2}$ Department of Obstetrics and Gynecology, University of Michigan Medical School, Ann Arbor, MI, USA

\section{${ }^{*}$ Correspondence}

Abel Teshome, Department of Obstetrics and Gynecology, St. Paul's Hospital Millennium Medical College, Addis Ababa, Ethiopia. Email: teshomeabela@gmail.com

Funding Information

Saint Paul's Hospital Millennium Medical College

\begin{abstract}
Objective: To assess the quality of family planning counseling among women attending a prenatal clinic in Addis Ababa, Ethiopia.

Methods: In a descriptive cross-sectional study conducted between February and April, 2015, at the prenatal care clinic of Saint Paul's Hospital Millennium Medical College, Addis Ababa, Ethiopia, pregnant women in their third trimester were interviewed about their experience of family planning counseling. Data were collected via a questionnaire. Logistic regression was used to assess predictors of satisfaction with the counseling service.

Results: During the study period, 400 women were interviewed. Only 139 women (34.8\%) were counseled about family planning. Among those counseled, 126 (90.6\%) decided to use a contraceptive method after delivery and 46 (36.7\%) decided to use an injectable contraceptive. Women were more likely to report high satisfaction when their provider asked about their partner's attitude toward contraceptive methods (adjusted odds ratio 6.6; $P<0.001$ ), and when asked about their concerns and worries regarding family planning methods (adjusted odds ratio $5.1 ; P<0.001$ ).

Conclusion: Very few women were counseled about contraception during prenatal care. Asking about a partner's attitude toward contraceptives and discussing women's fears or worries about contraceptives should be considered during family planning counseling to improve satisfaction and quality of care.
\end{abstract}

\section{KEYWORDS}

Contraceptive; Counseling; Family planning; Patient satisfaction; Prenatal care; Quality

\section{1 | INTRODUCTION}

According to the most recent Ethiopian Demographic and Health Survey (2011), the population of Ethiopia has increased steadily over the past 30 years and $25 \%$ of married women have an unmet need for family planning services. ${ }^{1}$ Improving family planning service in countries with high fertility rates has the potential to decrease poverty. ${ }^{2}$ Family planning is a way of improving the health of women and part of a strategy to decrease the high maternal mortality rate in Ethiopia. ${ }^{3}$

Family planning counseling is the process of giving information, clearing misunderstandings, and helping women to make an informed voluntary choice. ${ }^{3}$ The WHO has prepared and recommends different Information Education and Communication (IEC) tools to support family planning counseling. ${ }^{4}$ In low-income countries, use of family planning would decrease unplanned pregnancies by more than twothirds, prevent almost $70 \%$ of maternal deaths, and decrease unsafe abortions by $73 \%{ }^{5}$

Despite the above-mentioned benefits of family planning counseling and service provision, so far little research has been done to assess the quality of family planning counseling in Ethiopia. Furthermore, to our knowledge, no studies have assessed the quality of family planning counseling among women attending prenatal care, which is an ideal time 
to counsel women about family planning methods. ${ }^{3,6,7}$ The aim of the present study was therefore to assess the provision and quality of family planning counseling among pregnant women in Addis Ababa, Ethiopia.

\section{2 | MATERIALS AND METHODS}

The present cross-sectional study was conducted at the prenatal care clinic of Saint Paul's Hospital Millennium Medical College (SPHMMC), Addis Ababa, Ethiopia, among pregnant women in their third trimester between February 1 and April 30, 2015. Ethical approval of and clearance for the study was obtained from the Ethical Review Board of SPHMMC before the start of data collection. Informed consent was obtained from all women who were interviewed.

Saint Paul's Hospital Millennium Medical College is the second largest hospital in Ethiopia, and the prenatal care clinic takes referrals from eight satellite health centers in Addis Ababa, in addition to rural parts of Ethiopia. The prenatal care clinic has three stations and, on average, 100 women per day are seen in the clinic, which runs Monday to Friday from 8:00 AM to 12:30 PM. The prenatal care services are provided by obstetrics and gynecology residents.

All pregnant women in their third trimester who attended the clinic during the study period were eligible for inclusion. The exclusion criteria were emergency obstetric conditions, labor, first and second trimester of pregnancy, and ultrasound diagnosis of a lethal fetal congenital anomaly.

A systematic random sampling method was used for interview, whereby one in two women attending the clinic was interviewed. The sample size was calculated by using the Kish formula, $n=Z^{2} p(1-p) / w^{2}$, where $Z$ is $Z$-score, $p$ is percentage, and $w$ is width (precision). Because no previous research has been done in the study set-up, it was assumed that $50 \%$ of women would be counseled. Allowing for a precision of $5 \%$ and $95 \%$ confidence interval, a sample size of 385 women was calculated; with rounding, 400 women were interviewed about family planning methods.

Data were collected by means of an exit interview conducted at the prenatal clinic by nurses who were not working in the clinic. The questionnaire was modified from The Johns Hopkins School of Public Health Center for Communication Programs IEC Research Tools, translated into Amharic (the local language), and validated by back-translation into English. The quality of the data was assured by using this validated questionnaire, by training the data collectors and supervisor, and by pre-testing the data collection tools on $5 \%$ of the study women.

Participants who reported being counseled about family planning were asked a series of questions about what happened during this counseling, including whether their provider asked which method they wanted, if their spouse or partner approved of them using a family planning method, if their provider inquired about any fears or worries about using family planning, and if the provider acknowledged and responded to any concerns they had.

Note that "interest to use contraception methods" was recorded as the woman's interest when she was given option of choosing one or more methods of contraception; thus, she could choose more than one form of contraception. "Decision to use contraception method" was the woman's decision to use a contraceptive after she was given a choice from many forms of available methods; thus, she could choose only one form of contraception method.

A satisfaction score was used to measure the women's level of satisfaction with the service. Quality was measured in terms of patient satisfaction (measured by the patient's stated satisfaction of care), and the availability of the counseling service and family planning methods.

After the completeness and coding of questionnaires was checked, quantitative data were analyzed by using SPSS version 20 for Windows (IBM, Armonk, NY, USA). Data were reported as number (percentage) and a $P$ value of less than 0.05 was taken to be significant.

Logistic regression was used to evaluate factors associated with women's satisfaction with the counseling service. The independent variables were age, educational level, marital status, parity, and previous knowledge of contraception methods. The dependent (outcome) variable was level of satisfaction (high or low), which was dichotomized after data collection by using the median split of the satisfaction score.

\section{3 | RESULTS}

During the study period, 400 women attending the prenatal clinic were interviewed. Of these, 139 (34.8\%) had received family planning counseling, and their socio-demographic characteristics are shown in Table 1. The mean age of the counseled women was 28.45 years, and

TABLE 1 Socio-demographic characteristics of women attending prenatal care who were counseled about family planning methods. ${ }^{a}$

\begin{tabular}{|lc|}
\hline Characteristic & Value \\
\hline Age group, y & \\
\hline $15-19$ & $3(2.2)$ \\
\hline $20-24$ & $23(16.5)$ \\
\hline $25-29$ & $60(43.2$ \\
\hline $30-34$ & $35(25.2)$ \\
\hline$\geq 35$ & $18(12.9)$ \\
\hline Age, y & $4.80(18-42)$ \\
\hline Educational level & \\
\hline None & $17(12.2)$ \\
\hline Primary & $48(34.5)$ \\
\hline Secondary & $53(38.1)$ \\
\hline University/college & $21(15.1)$ \\
\hline Religion & \\
\hline Orthodox Christian & $81(58.3)$ \\
\hline Muslim & $41(21.5)$ \\
\hline Protestant Christian & $17(12.2)$ \\
\hline Marital status & $2(1.4)$ \\
\hline Married & $2(1.4)$ \\
\hline Single & $135(97.1)$ \\
\hline Divorced & \\
\hline
\end{tabular}

${ }^{a}$ Values are given as mean $\pm \mathrm{SD}$ (range) or number (percentage). 
$60(43.2 \%)$ were in the age range $25-29$ years. Many of the women had completed secondary education $(53 ; 38.1 \%)$, and most were married (135; 97.1\%).

Among the 139 women counseled for family planning methods, 125 (89.9\%) expressed interest in using a contraceptive method when they were given the option to choose one or more methods. Overall, 92 (73.4\%) expressed an interest in using injectable contraceptives, 67 (53.2\%) in using an intrauterine device, 58 (46\%) in using condoms, 57 (45.3\%) in using pills, 44 (35.3\%) in using the implant, and 35 (28\%) in using natural family planning methods (Fig. 1).

Figure 2 summarizes the information given to the pregnant women. Among the 139 counseled women, 110 (77.7\%) were informed about the implant, 110 (77.7\%) about the intrauterine device,

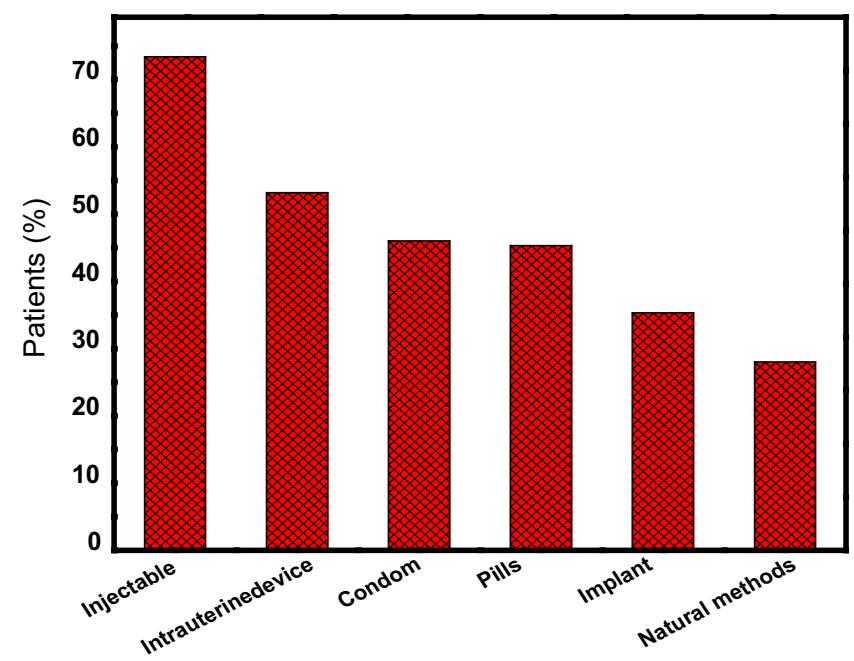

Types of contraception

FIGURE 1 Types of contraceptive method that prenatal women expressed an interest in using after family planning counseling at Saint Paul's Millennium Medical College, Addis Ababa, Ethiopia.

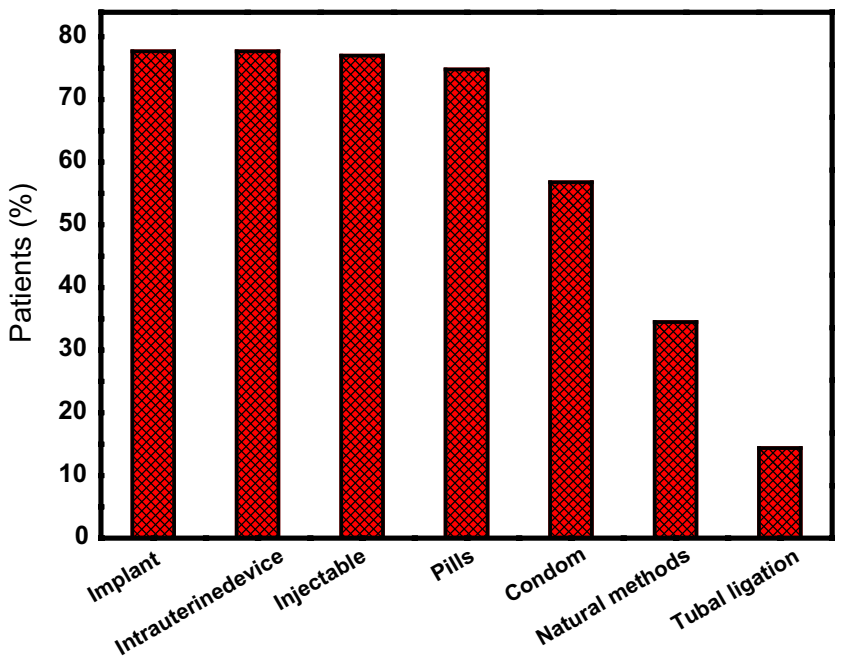

Types of contraception

FIGURE 2 Types of contraceptive method that prenatal women were informed about during the family planning counseling service at Saint Paul's Millennium Medical College, Addis Ababa, Ethiopia.

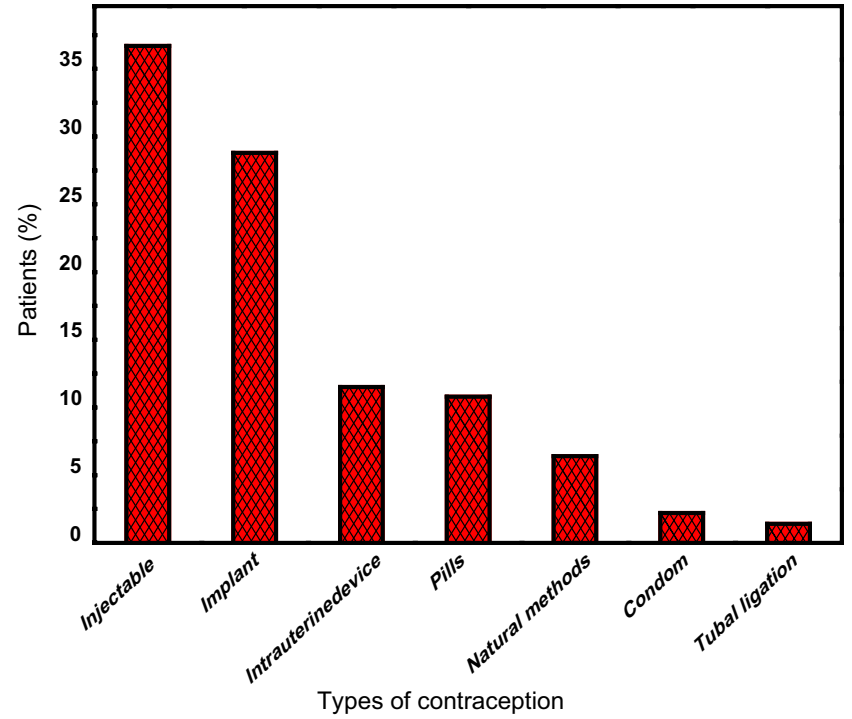

FIGURE 3 Types of contraceptive method that prenatal women decided to use after receiving the family planning counseling service at Saint Paul's Millennium Medical College, Addis Ababa, Ethiopia, January.

107 (77\%) about the injectable contraceptive, 104 (74.8\%) about pills, 79 (56.8\%) about condoms, 48 (34.5\%) about natural family planning, and 20 (14.4\%) about tubal ligation.

Regarding the decision to use one form of contraception method after counseling, 126 (90.0\%) of counseled women decided to use a contraception method after delivery. Of these, 46 (36.7\%) decided to use the injectable contraceptive, $36(28.8 \%)$ to use the implant, 15 (11.5\%) to use an intrauterine device, $14(10.8 \%)$ to use pills, $8(6.4 \%)$ to use natural family planning, $3(2.2 \%)$ to use condoms, and 4 (3.4\%) to use tubal ligation (Fig. 3).

In terms of satisfaction, 133 (95.7\%) of the women reported that they were satisfied with the counseling service that they had received. No woman reported being counseled by means of IEC materials (such as flip charts, anatomic models, or contraceptive samples).

A reliability analysis was carried out on 11 questions about satisfaction that were answered on a five-point Likert scale with score values ranging from 1 (strongly disagree) to 5 (strongly agree) to determine whether these variables could be used to assess satisfaction. All questions were found to be valid with a Cronbach $\alpha$ value of 0.8 or higher (Table 2).

The satisfaction score for the 11 questionnaire variables ranged from 33 to 55. The mean and median for the satisfaction score were both 47 and the satisfaction score was found to be normally distributed (Fig. 4). A dependent dichotomous variable was created by the median split. Those who scored below the median were assigned to have low satisfaction, and those who score above the median were assigned to have high satisfaction. Bivariate and multivariate regression analysis was used to assess the relationship between the women's satisfaction and independent variables.

Bivariate analysis indicated that a woman was 4.2 times more likely to have high satisfaction when she had previous knowledge 
TAB LE 2 Reliability analysis of variables used to assess satisfaction.

\begin{tabular}{|c|c|c|c|}
\hline Variable & Scale mean if item deleted & Scale variance if item deleted & Cronbach $\alpha$ if Item deleted \\
\hline Would recommend provider & 42.6475 & 17.041 & 0.818 \\
\hline Competency of provider & 42.5827 & 17.448 & 0.470 \\
\hline Harm would not be done by provider & 42.5827 & 17.259 & 0.819 \\
\hline Trustful of provider with secret & 42.5755 & 17.246 & 0.814 \\
\hline Clinic site easy to reach & 42.6691 & 15.687 & 0.806 \\
\hline Waiting time not too long & 43.1007 & 15.280 & 0.825 \\
\hline Clean clinic area & 42.6906 & 16.708 & 0.813 \\
\hline Sufficient information given & 42.6835 & 16.218 & 0.810 \\
\hline Privacy maintained & 42.6691 & 16.513 & 0.809 \\
\hline Sufficient time for discussion & 42.5324 & 15.642 & 0.801 \\
\hline Friendly staff at service provider & 42.4748 & 16.976 & 0.813 \\
\hline
\end{tabular}

about contraception methods (odds ratio [OR], 4.2; 95\% confidence interval $[\mathrm{Cl}], 1.1-16 ; P=0.038$ ) (Table 3). In addition, those who were previously interested in using a specific contraception method were 3.5 times more likely to have high satisfaction in the family planning service (OR, 3.5; 95\% Cl, 1-11; $P=0.042$ ). Women who got encouragement from the family planning counselor were 3.8 times more likely to express high satisfaction for the counseling session (OR, 3.8; $95 \% \mathrm{Cl}$, 1.3-11; $P=0.016$ ). Age, educational level, marital status, and a woman's need to have more children were not significantly associated with level of satisfaction.

Multivariate analysis showed that women were 6.6 times more likely to have high satisfaction when their provider asked about their partner's attitude toward contraceptive methods (adjusted OR [AOR], 6.6; 95\%, Cl, 2.5-8.0; $P<0.001$ ) (Table 4). Women whose provider asked about their concerns and worries regarding family planning contraceptive methods were five times more likely to express a high level of satisfaction (AOR, 5.1; 95\% Cl, 2.3-11; $P<0.001$ ). Women were 4.7 times more likely to have a high level of satisfaction when the healthcare provider acknowledged their concerns about family planning (AOR, 4.7; 95\% Cl, 2.1-10.4; $P<0.001$ ). If the healthcare provider did not recommend a specific contraception method for the woman, the woman was 17.7 times more likely to have a high level of satisfaction when the reason for not recommending a specific method was discussed by the healthcare provider (AOR, 17.7; 95\% Cl, 7.0-10.4; $P<0.001)$.

\section{DISCUSSION}

The present findings showed that only 139 (34.8\%) of women were counseled about family planning during prenatal care. Of those who were counseled, the majority showed interest in using contraceptive

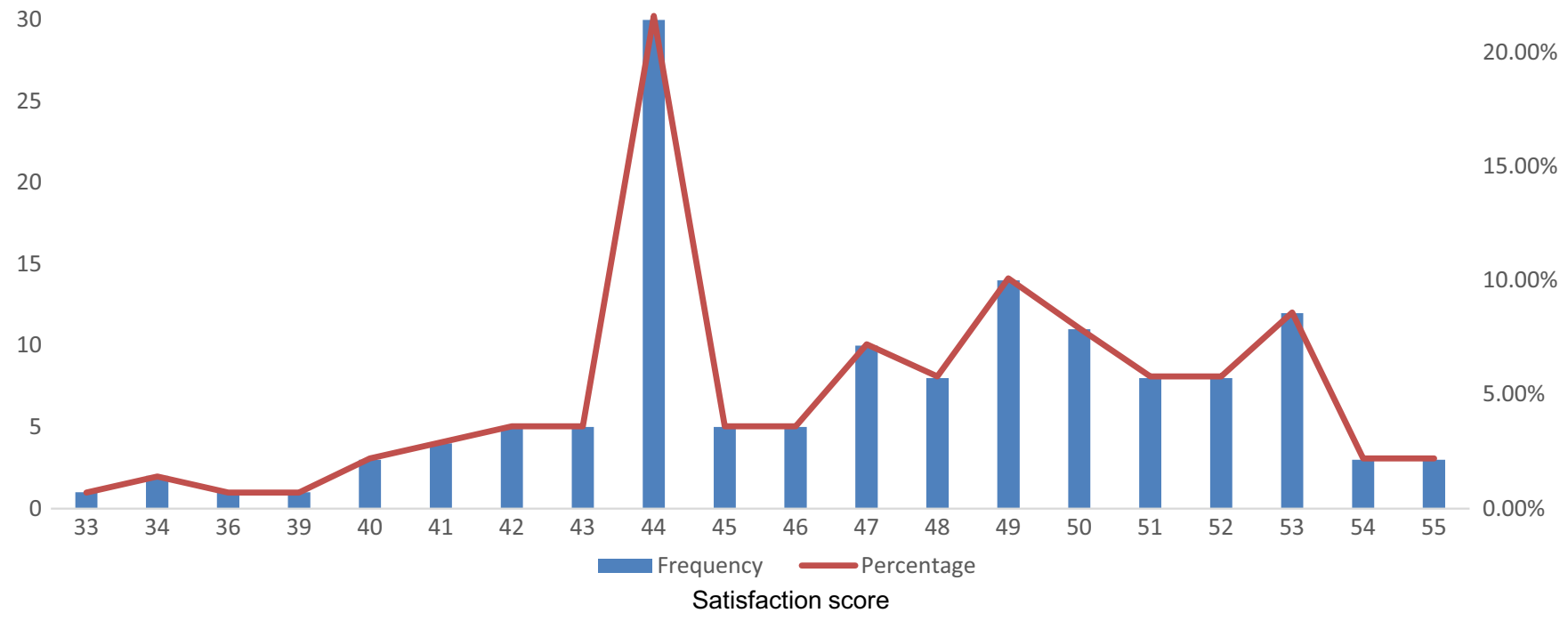

FIGURE 4 Distribution of the frequency of satisfaction score. 
TAB LE 3 Bivariate logistic regression for predictors of satisfaction with the family planning counseling service.

\begin{tabular}{|lcc|}
\hline Variable & OR (95\% Cl) & P value \\
\hline Previous knowledge of patient $^{\mathrm{a}}$ & $4.2(1.1-16.0)$ & 0.038 \\
\hline Patient's interest $^{\mathrm{b}}$ & $3.5(1.0-11.0)$ & 0.042 \\
\hline Asked about partner's attitude $^{c}$ & $7.5(2.8-20.0)$ & $<0.001$ \\
\hline Asked about patient's concerns $^{\mathrm{c}}$ & $5.1(2.3-11.0)$ & $<0.001$ \\
\hline Acknowledged patient's concerns $^{c}$ & $7.8(3.5-17.5)$ & $<0.001$ \\
\hline $\begin{array}{l}\text { Discussed reasons for not } \\
\text { recommending a contraceptive }^{\text {method }} \mathrm{c}\end{array}$ & $18.5(7.6-45.0)$ & $<0.001$ \\
\hline Encouraged patient $^{\mathrm{c}}$ & & \\
\hline Age & $3.8(1.3-11.0)$ & 0.016 \\
\hline Educational level & $0.8(0.5-1.1)$ & 0.146 \\
\hline Marital status & $0.8(0.9-1.9)$ & 0.500 \\
\hline Need for more children & $1.0(0.1-7.2)$ & $>0.99$ \\
\hline
\end{tabular}

${ }^{a}$ About contraceptive methods.

${ }^{\mathrm{b}}$ In a specific contraceptive method.

${ }^{\mathrm{C}}$ By healthcare provider (family planning counselor).

methods when they were given an option to choose one or more methods: injectable contraceptive was the preferred method for most women. Most women were informed about long-term contraceptive methods (implant and intrauterine device) in accordance with the Ministry of Health guidelines, ${ }^{3}$ but when it came to the decision to use contraception after delivery, many decided to use the injectable contraceptive. This result is consistent with findings in the Ethiopian Demographic Health Survey 2011. ${ }^{1}$

The advantage of quality of care in provision of family planning services is widely recognized; therefore, there is a need to develop a simple means of assessing this quality. Of particular interest are approaches that take into consideration women's satisfaction with their care. ${ }^{8}$ In the present study, information related to the quality of family planning counseling services was obtained by means of an exit interview at the prenatal clinic in Saint Paul's Hospital Millennium Medical College.

A study in Nigeria reported that the prenatal clinic was a major source of information on contraceptive methods. ${ }^{9}$ In the present study, however, only $35 \%$ of women interviewed had been counseled for family planning methods. A possible explanation for this difference might be that the healthcare providers were overwhelmed by the high number of women visiting the prenatal clinic or requiring other obstetric care; nevertheless, this represents a missed opportunity for family planning counseling.

No woman in the current study was counseled by means of IEC materials (e.g., flip charts, anatomic models, or contraceptive samples). Similarly, in a study in northwestern Ethiopia, none of the family planning service providers used IEC materials during counseling. ${ }^{10}$ Although it is recommended that these materials should be used, they are not available at prenatal care clinics in Ethiopia.

Overall, 133 (95.7\%) of the 139 counseled women reported that they were satisfied with the counseling service that they had received. This finding is consistent with that of a study in public health centers in Jimma, Ethiopia, where $93.7 \%$ of women reported satisfaction with the service they had received. ${ }^{11}$ This high level of satisfaction might be due to courtesy bias, which is a limitation of this type of study. Although the majority reported being satisfied overall, when satisfaction was assessed differently via the satisfaction score, the even distribution of these scores suggested that not all women were satisfied.

In the present study, women were more likely to express a high level of satisfaction when the provider asked about their concerns and worries regarding family planning methods (AOR, 5.1; $P<0.001$ ); when the healthcare provider acknowledged their concerns about family planning methods (AOR, 4.7; $P<0.001$ ); and in cases where the healthcare provider did not recommend a specific contraception method, when they discussed the reasons why (AOR, 17.7; $P<0.001$ ).

Patient age, marital status, and need for more children were not found to be significant predictors of satisfaction with the counseling service. Again, these findings are consistent with those of the study in Jimma, Ethiopia. ${ }^{11}$ A study in Malawi, the Malawi Male Motivator Project, found that ease of communication with couples and involvement of males during contraceptive counseling were the only predictors of contraceptive uptake. In the present study, women more likely to be satisfied when they are asked about their partner's attitude toward contraceptive methods during counseling (AOR, 6.6; $P<0.001) .{ }^{12}$

The study has potential limitations. First, the women might have considered that their prenatal service would be compromised on their next visit if they expressed negative feelings about the counseling service (i.e., courtesy bias), which is inherent in this type of study. Second, there is no standard satisfaction score to classify the quality of the counseling service; therefore, the satisfaction score median was used as a cut-off to classify the satisfaction as high versus low. Last, there might have been some modification of behavior by the healthcare providers during the study period (whereby they improved their quality of contraceptive counseling because they felt their performance was

TAB LE 4 Multivariate logistic regression for predictors of satisfaction with the family planning counseling service.

\begin{tabular}{|c|c|c|}
\hline Variable & Adjusted odds ratio ( $95 \%$ confidence interval) & $P$ value \\
\hline Asked about patient's concerns about family planning ${ }^{a}$ & $5.1(2.3-11)$ & $<0.001$ \\
\hline Reasons discussed for not recommending a specific contraceptive method ${ }^{a}$ & $17.4(7.0-10.4)$ & $<0.001$ \\
\hline
\end{tabular}

${ }^{\mathrm{a} B y}$ healthcare provider (family planning counselor). 
being monitored), a phenomenon known as the Hawthorne effect. However, this effect is more commonly seen in studies that collect data by the direct observation of service provision. In addition, a systematic random sampling technique was used to recruit women for the exit interview to minimize the Hawthorne effect.

In conclusion, the provision of family planning counseling to pregnant women attending a prenatal clinic was found to be low. Most women were counseled for long-term contraceptive methods, but a high number decided to use the injectable contraceptive. Asking about a partner's attitude, asking about the women's concerns about using contraception methods, and acknowledging the women's concerns were found to be significant predictors of satisfaction with the counseling service. Involving the male partner and improving the interpersonal skills of providers need to be considered during family planning counseling.

\section{AUTHOR CONTRIBUTIONS}

AT contributed to the study design, data collection and analysis, and writing the manuscript. MB contributed to study planning, data analysis, and to editing and reviewing the manuscript. SDR contributed to study planning, literature searches, and to editing and reviewing the manuscript.

\section{ACKNOWLEDGMENTS}

The study was funded by Saint Paul's Hospital Millennium Medical College.

\section{CONFLICT OF INTEREST}

The authors have no conflicts of interest.

\section{REFERENCES}

1. Ethiopia Demographic and Health Survey 2011. Published 2012. http://gdtc.bdu.edu.et/sites/default/files/Documents/Ethiopia_ Demographic_health_Survey_2011.pdf. Accessed January 24, 2017.

2. John Cleland SB. Alex Ezeh, Anibal Faundes, Anna Glasier, Jolene Innis. Family planning: The unfinished agenda. Lancet. 2006;368:1810-1827.

3. Federal Republic of Ethiopia, Ministry of Health. National Guideline for Family Planning services in Ethiopia. 2011.

4. World Health Organization. Information, Education and Communication. 2001.

5. Susheela S, Darroch JE, Ashford LS, Vlassoff M. Adding It Up: The Costs and Benefits of Investing in Family Planning and Maternal And Newborn Health. Guttmacher Institute and UNFPA; 2009.

6. Federal Republic of Ethiopia, Ministry of Health. Management protocol on selected obstetrics topics. 2010.

7. Barber SL. Family Planning Advice and Postpartum Contraceptive Use Among Low-Income Women in Mexico. Int Fam Plan Perspect. 2007;33:6-12.

8. Timothy Williams JS-AaYC. Measuring Family Planning Service Quality through Client Satisfaction Exit Interviews. Int Fam Plan Perspect. 2000;26:63-71.

9. Allagoa DO, Nyengidiki TK. Knowledge, Attitude And Practice Of Contraception Amongst Antenatal Patients At The University Of Port Harcourt Teaching Hospital, Port Harcourt. Nigerian Health J. 2011;11:89-90.

10. Fantahun M. Quality of family planning services in northwest Ethiopia. Ethiop J Health Dev. 2005;19:195-2002.

11. Tafese F, Woldie M, Megersa B. Quality of family planning services in primary health centers of Jimma Zone, South West Ethiopia. Ethiop J Health Sci. 2013;23:251-253.

12. Shattuck D, Kerner B, Gilles K, Hartmann M, Ng'ombe T, Guest G. Encouraging contraceptive uptake by motivating men to communicate about family planning: The Malawi Male Motivator Project. Am J Public Health. 2011;101:92-93. 